\title{
Longer bars for bigger numbers? Children's usage and understanding of graphical representations of algebraic problems
}

\author{
Kerry Lee, Kiat Hui Khng, Swee Fong Ng, Jeremy Ng Lan Kong
}

National Institute of Education, Nanyang Technological University, Singapore

Article received 7 June 2013 / revised 19 July 2013 / accepted 16 August 2013 / available online 27 August 2013

\begin{abstract}
In Singapore, primary school students are taught to use bar diagrams to represent known and unknown values in algebraic word problems. However, little is known about students' understanding of these graphical representations. We investigated whether students use and think of the bar diagrams in a concrete or a more abstract fashion. We also examined whether usage and understanding varied with grade. Secondary $2\left(N=68, M_{\text {age }}=13.9\right.$ years $)$ and Primary 5 students $\left(N=110, M_{\text {age }}=11.1\right.$ years $)$ were administered a production task in which they drew bar diagrams of algebraic word problems with operands of varying magnitude. In the validation task, they were presented with different bar diagrams for the same word problems and were asked to ascertain, and give explanations regarding the accuracy of the diagrams. The Küchemann algebra test was administered to the Secondary 2 students. Students from both grades drew longer bars to represent larger numbers. In contrast, findings from the validation task showed a more abstract appreciation for how the bar diagrams can be used. Primary 5 students who showed more abstract appreciations in the validation task were less likely to use the bar diagrams in a concrete fashion in the production task. Performance on the Küchemann algebra test was unrelated to performance on the production task or the validation task. The findings are discussed in terms of a production deficit, with students exhibiting a more sophisticated understanding of bar diagrams than is demonstrated by their usage.
\end{abstract}

Keywords: Algebra; Pre-algebra; Graphical representation; Mathematical understanding 


\section{Introduction}

Singapore has performed well in recent international tests of mathematics (Mullis, Martin, Gonzalez, \& Chrostowski, 2004; OECD, 2010). Perhaps because of this, there has been much interest in the Singapore mathematics curriculum, with some schools in other countries having been reported to have adopted her curriculum (e.g., $\mathrm{Hu}, 2010)$. The wisdom of such cross-country adoption aside, one peculiar feature of the Singapore curriculum is that algebraic thinking is introduced early. Unlike many countries where algebra is introduced in the secondary or high school years, mathematical problems with an algebraic structure are taught in the senior primary years (Grades $4-6$ ).

Algebra is recognised widely as an important pillar for both academic and economic success (National Council for Teachers of Mathematics, 2000; National Mathematics Advisory Panel, 2008). Primary school children have been shown capable of exhibiting algebraic thinking (e.g. Carpenter \& Levi, 2000; Carraher, Schliemann, Brizuela, \& Earnest, 2006; Ng \& Lee, 2009; Swafford \& Langrall, 2000; Warren \& Cooper, 2005; Warren \& Cooper, 2009). However, whether this understanding is similar to that of older students has not been studied widely. Certainly, algebra can be difficult; even for college students. Some of the documented difficulties include intrusion of arithmetic reasoning (e.g. Khng \& Lee, 2009; Ng, 2003; Stacey \& MacGregor, 1999), difficulties translating word problems to equations (e.g. Capraro \& Joffrion, 2006; Duru, 2011; Hefferman \& Koedinger, 1997), problems with the concept of equivalence (e.g. Hunter, 2007; Kieran, 1981; Knuth, Stephens, McNeil, \& Alibali, 2006; Steinberg, Sleeman, \& Ktorza, 1991) and poor understanding of the concept of variables (e.g. Küchemann, 1978).

In this study, we focused on an important pedagogical device for providing children with earlier access to algebraic problems. In the latter part of Primary 4 (Grade $4, \sim 10$ years old), children in Singapore are introduced to algebraic or start-unknown word problems. Instead of symbolic algebra, children are taught a graphical heuristic in which they draw bar diagrams to represent known and unknown quantities $(\mathrm{Ng} \&$ Lee, 2005). We examined children's usage and understanding of this heuristic.

\subsection{An early start to learning algebra}

This graphical heuristic, also called the model method, provides students with access to problems that would otherwise require symbolic algebra. Three different types of graphical models are commonly taught: (a) part-whole, (b) comparison, and (c) multiplication and division models ( $\mathrm{Ng} \&$ Lee, 2009). To illustrate its operation, take for example a simple question.

Mary and John have 6 marbles altogether. John has 2 more marbles than Mary. How many marbles does Mary have?

Figure 1 shows the graphical and letter symbolic approach to the problem. In the graphical approach, students draw rectangular bars to represent the number of marbles carried by Mary and John. The difference between the two quantities is shown by drawing one bar longer than the other. Because the quantitative difference is specified in the question, John's bar is drawn longer and the quantity represented by the difference in length -- the difference unit -- is labelled as 2 . With this graphical representation, children typically proceed with a variety of arithmetic strategies, such as unwinding or guess-and-check (Nathan \& Koedinger, 2000), to arrive at the solution. 


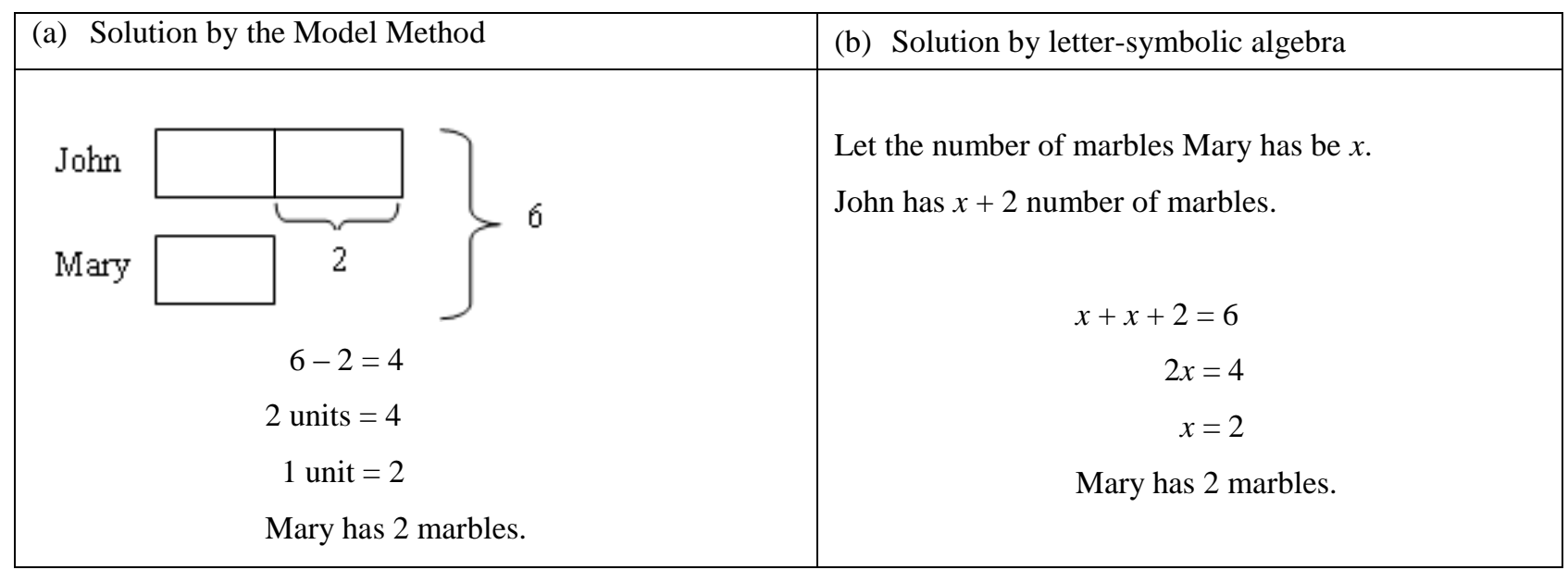

Figure 1. Model method and the symbolic algebra approach to the question "Mary and John have 6 marbles altogether. John has 2 more marbles than Mary. How many marbles does Mary have?" The bar labelled "2" in (a) represents the difference unit.

Formal algebraic notations in the form of letter symbols and related expressions were not introduced till some time into Grade 6. Although both the model method and symbolic algebra require students to translate information in a word problem to an alternative representation, there are some fundamental differences. Symbolic algebra requires students to work directly with unknown quantities. An equation comprising both known and unknown values is formed using forward operations and the unknown is solved by constructing a series of equivalent expressions. Students operate on the equation in a way that maintains symmetric equivalence across the two sides of the equals sign.

In contrast, the graphical approach can trace its roots to pedagogical tools used in the early primary school years. Beginning in lower primary, familiar objects and pictures (e.g., pictures of bears and dolls) are used to depict known quantities as an aid to understanding arithmetic word problems. With the graphical approach, a standardized representational tool is used to represent both known and unknown quantities. Of course, unknown quantities cannot be depicted exactly. Instead, children are taught to draw a diagram with the unknown quantities depicted by bars of arbitrary length, which are constrained by the given quantities and their quantitative relations. Computation of solution is effected using arithmetic procedures in which students work only with known quantities. In other words, the unknown is solved by the direct application of arithmetic operations on known values (e.g., backward operations such as unwinding). Unlike symbolic algebra, with the graphical approach, the equals sign is generally used as a directive to calculate instead of representing equality (see Khng \& Lee, 2009, for more details).

Some recent works on the use of the model method were motivated by parents' and teachers' concerns that the model method may confuse students when it comes time for them to learn letter symbolic algebra, with some concerned that the two approaches may draw on different cognitive processes (e.g., Kwokwc, 2011; Lim, 2007). The model method is taught system-wide and has been part of the national curriculum in Singapore for over a decade. For this reason, it is difficult to evaluate these claims using standard programme evaluation methodology. In two recent studies, Lee and his colleagues used functional magnetic resonance imaging techniques to examine the cognitive underpinnings of these two approaches (Lee et al., 2007; Lee et al., 2010). The findings showed substantial overlap between the two approaches, but symbolic algebra activated more strongly areas associated with attention and working memory engagement. These findings suggest that symbolic algebra is more demanding of cognitive resources than the model method. From a pedagogical viewpoint, introducing the model method prior to symbolic algebra can thus be interpreted as being consistent with their respective cognitive demands. 


\subsection{The model method, letter Symbols, and variables}

Regarding the cognitive factors that influence children's success in algebra, there has been a number of recent studies on both domain-general and domain-specific correlates of algebraic performance (e.g., Fuchs et al., 2012; Lee, Ng, Bull, Pe, \& Ho, 2011; Tolar, Lederberg, \& Fletcher, 2009; Wei, Yuan, Chen, \& Zhou, 2012). On the specific influence of using diagrams, there is a large body of research that examined whether students learn better when text is accompanied by diagrams (Mayer, 1989, 2002) or when students generate the diagrams themselves (Meter \& Garner, 2005). Of particular relevance are a number of studies conducted by Koedinger and his colleagues. They investigated the use of "picture algebra", a strategy similar to the model method. They found that by using this strategy, even students in Grade 6 were successful in solving algebraic problems that are known to be challenging for older students (Koedinger \& Terao, 2002). The strategy was also found to be effective for lower achieving pre-algebra students (Booth \& Koedinger, 2010).

Although we now have some information on the efficacy of the model method and some of the contexts in which they are more likely to be efficacious, an important issue on which we know little is how students understand or perceive these graphical representations. Compared to pedagogical practices in earlier grades in which familiar objects are used to depict operands in arithmetic in a one-to-one manner, the model method involves a greater degree of abstraction. Instead of familiar and discrete objects (e.g., bears or dolls), bars of different lengths are used. Nonetheless, given students' earlier experiences, it is possible that they retain a concrete way of thinking about the bars, such that a bar of a certain length is deemed capable of holding, say, ten bears and ten bears alone. In teaching the model method, teachers generally ask children to use relatively longer bars for bigger numbers ( $\mathrm{Ng} \& \mathrm{Lee}, 2009$ ). More important than absolute length is that, within a problem, children are taught that the lengths of the bars should preserve the quantitative relations between the protagonists. This is especially when more than two protagonists are involved in a problem.

How students understand these graphical representations is important because when they learn symbolic algebra, a concept that they should understand is that letter symbols (e.g., $x$ and $y$ ) denote variables. Although the model method gives children earlier access to algebraic questions without the use of letter symbols, the bars that are used to depict quantities play a similar role as do letter symbols in algebraic equations. Both serve to depict relations between known and unknown quantities. If children regard the bar diagrams in a concrete manner, one potential drawback is that they may over-generalise and regard $x$ and $y$ as depicting unknown constants. A related concern was raised by Dede (2004), who argued that the preponderance of questions that use letter-symbols to represent unknowns could result in students developing a restricted view of the roles and functions of variables.

The concept of a variable is important in algebra, but it is also difficult, perhaps because it has different meanings or usages. Usiskin (1988) argued that variables are used in different ways: (a) as an unknown or a constant, (b) a pattern generalizer, (c) an argument or parameter, or (d) an arbitrary mark on paper. When students are first introduced to letter symbols, they typically encounter them as unknowns in solve-for- $x$ questions, where students are asked to find a solution for the letter symbols (e.g., $x+y=47, x+$ $13=y$. What is the value of $x$ ?). As Dede (2004) argued, one concern is that given the preponderance of experiences with this usage of letter symbols, children come to take this as the norm and neglect to entertain other ways in which variables are used. Indeed, when asked to simplify algebraic expressions (e.g., $3 x+5 x-$ 24), children tended to find a solution for $x$ instead (Philipp, 1992). A similar difficulty was reported by Akgün and Özdemir (2006). In their study, students presented with $x+2=2+x$ attempted to solve for $x$ when they were told to report all the values that $x$ can assume. Kuchëmann (1978) investigated students' understanding of the use of letter symbols and found most secondary school students treated them as concrete placeholders or "shorthand names" (MacGregor \& Stacey, 1997) (e.g., $p$ in 3p as pears instead of number of pears). Only a small number of students displayed an understanding of letters as representing specific unknowns. An even smaller number considered the letters to be generalized numbers. 


\subsection{The present study}

To understand better the utility of teaching the method model, we focused on students' understanding of these graphical representations. Specifically, we investigated whether students use and think of the bar diagrams in a concrete or a more abstract fashion. We asked children to perform two tasks. In the production task, we examined how they drew model representations for algebraic word problems with operands of varying quantities. We defined concrete usage as varying the length of bars across questions in accordance to the magnitude of operands. Abstract usage was indicated by the lack of a consistent relation between the length of the bars and the magnitude of operands. We also manipulated the sequence in which increases in the magnitude of operands were presented. Because a sequential increase may overly focus the children's attention on changes in magnitude and attenuate any tendency to adopt a more abstract strategy, we also presented the increases in a random sequence. In the validation task, we assessed the same children's understanding by asking them to judge and explain whether several presented graphical representations were drawn correctly. The representations contained drawings that fall into either the concrete or abstract pattern as defined above. Children with more sophisticated understanding were expected to know that though bars of particular lengths depict unknown constants within the context of each question, across questions, the same bars can be used to depict different quantities. In fact, it is when letter symbols are considered in this sense that they are considered variables.

We tested Primary 5 (Grade 5) children who have not been taught symbolic algebra and Secondary 2 (Grade 8 ) children who have been taught both the model method and symbolic algebra. The primary school children would have used the model method for a year, whereas the secondary school children would have been introduced to symbolic algebra some time during since Primary 6. In an interview study conducted with ten Primary 5 students, most of the children showed some abstract understanding of how the bar diagrams should be used (Ng \& Lee, 2008). Typical of their responses was statements suggesting that the absolute size of the bars do not matter. With their added experience with algebraic problems, we expected the secondary school children to display more abstract usage and awareness. To examine how our measures of children's understanding were related to other tests of algebraic understanding, we administered the Küchemann algebra test (Brown, Hart, \& Kuchemann, 1985) to the secondary school children. This is a standardized measure that gauges children's understanding of variables expressed in the letter symbolic format. It was given only to secondary school children as the primary school children have not been exposed to letter symbols.

\section{Method}

\subsection{Participants and design}

The experiment was based on a 4 (Magnitude band: one, tens, hundreds, versus thousands) $\times 2$ (Question sequence: increasing value versus randomised) $\times 2$ (Grade: Primary 5 versus Secondary 2 ) full factorial split-plot design. Magnitude band served as the only within-subject variable. A total of 68 Secondary 2 students $\left(M_{\text {age }}=13.9, S D=0.45\right)$ and 110 Primary 5 students $\left(M_{\text {age }}=11.1, S D=0.60\right)$ from 5 schools (2 Secondary and 3 Primary) of mixed abilities and social economic status participated in the study. All schools were government funded, located in the western region of Singapore, and followed the national mathematics curriculum. All children participated with parental consent.

\subsection{Task and materials}

We designed a web-based program comprising a production task followed by a validation task. Participants' inputs on the computer interface were logged on a server. In addition, the Secondary 2 students were administered the Küchemann Algebra Test (Brown et al., 1985). 


\subsubsection{Production task}

The children were presented with algebra word problems on the computer screen and were asked to draw model diagrams for these problems using the graphical tools provided onscreen. A computerized interface provided a standardized interface both for problem administration and data collection. The children started with an online palette that contained a variety of specially designed drawing and labelling tools. The children viewed and worked on one problem at a time. They were not required to solve the problem, just to draw the bar diagrams in the same format that they would with pen and paper. There was no limit to the size of the bars that could be drawn. Each child completed 12 problems. All the problems were of the same structure, but varied in the name of the protagonists and referents (e.g., Mary versus Jane, marbles versus cupcakes).

The specific magnitude band in each problem varied across four scales: ones, tens, hundreds, and thousands. Three problems were given within each scale. The size of the operand relating to the difference unit -- our dependent measure of interest -- in these three problems was drawn from the smaller, medium, and larger range of each magnitude band; with one question from each range. In Figure 1a, for example, we depicted a question with a small difference unit ("2") from the magnitude band of "ones". A question with a large difference-unit operand (e.g., 7), still from the magnitude band of ones, would read something like "Mary and John have 9 marbles altogether. John has 7 more marbles than Mary..." A question with a large difference unit (e.g., 70) from the magnitude band of tens would have been presented as "John has 70 more marbles than Mary..."

To reduce stimuli specific effects, we developed two parallel sets of problems that were identical in structure and differed only in specific quantities. In both sets, questions were administered either in randomized order or in accordance to the magnitude of the difference-unit operands. The children's drawings were recorded by the computer program, which also logged the pixel length of the bar diagrams. The pixel length of the difference unit or the section of the bar that represents the difference between the two protagonists (see Figure 1a) was used as the dependent variable. Of particular interest was whether students varied the length of the difference unit across magnitude bands. That is, did they draw bars that were much longer to represent "John has 70 more marbles" as compared to "John has 7 more marbles"? Because we did not impose an upper limit on the length of the bar, there was also no upper cap on the range for the dependent variable. In Table 1, we provided both the confidence intervals and the range from the observed data.

\subsubsection{Validation task}

Participants were presented with two sets of 3 questions. For the first set of validation questions, each question comprised a word problem followed by two model diagrams differing in whether proportionality in the length of the bars was maintained, across questions. In one diagram, the bars were drawn with longer bars for larger numbers. In the other, proportionality was not maintained. In other words, the diagrams differed in whether the bars were drawn in a more concrete or abstract manner. Participants were asked to choose which diagram (one or the other, or both) was correct. They were also asked to explain their selection by selecting a response from four multiple choice options (see Appendix A).

For the second set of questions, each question contained two word problems, each accompanied by two diagrams, said to be drawn by Student A and Student B respectively. The two word problems in each question were structurally equivalent, differing only in the magnitude of the operands. Student A's diagrams demonstrated a more abstract usage of the bars: the size of the bars were identical for operands of different sizes. Student B's diagrams demonstrated a more concrete usage: the size of the bars differed in accordance to changes in the size of the operands. Participants were asked to indicate if the two students were correct. A maximum of 9 marks could be obtained in the validation task (see Appendix A for scoring criteria) with higher marks indicating a more abstract understanding of the bar diagrams. 


\subsubsection{Küchemann Algebra Test}

The Küchemann Algebra Test from the Chelsea Diagnostic Mathematics Tests (Brown et al., 1985) is a standardized measure of how children interpret letter symbols used in algebra. Up to six different common interpretations have been identified in the literature: (i) letter numerically evaluated, (ii) letter not used or ignored, (iii) letter used as an object or abbreviation, (iv) letter used as a specific unknown, (v) letter used as a generalised number, (vi) letter used as a variable. The test places children on one of four levels of understanding based on the type and complexity of their interpretations. For example, a student using any of the first three interpretations and can only answer very simple questions is classified as Level 1. A student using the same interpretations, but who is able to answer more structurally complex questions is classified as Level 2. Understanding letter symbols as referring to specific unknowns qualifies classification at Level 3. This is deemed a basic level of understanding required for symbolic algebra. Level 4 requires students to demonstrate an understanding of letter symbols as generalised numbers. Of interest was whether Secondary 2 students' attainment on the Küchemann test correlated with their performance on the production task. That is, did students with higher scores on the Küchemann test show less tendency to adjust the length of the bars in accordance to the magnitude of the operands?

\subsection{Procedure}

For the computerised tasks, participants from the same school were tested together in a single group session in their school computer laboratories. The Secondary 2 students completed the Küchemann Algebra Test in an additional session in a classroom.

\section{Results}

To examine whether children's performances on the production task differed across the various experimental conditions, we subjected the data to a 4 (Magnitude band: ones, tens, hundreds, versus thousands) $\times 2$ (Question sequence: increasing value versus randomised) $\times 2$ (Grade: Primary 5 versus Secondary 2) repeated measures multivariate analysis of variance. Pixel length of the difference unit drawn for questions with smaller, medium, versus larger operands within each magnitude band served as the dependent measures. In addition to the main independent variables, we entered which of the two parallel forms children were administered to take account of potential differences in performance across the two forms. Descriptive statistics can be found in Table 1. 
Table 1

Mean pixel length, standard deviation, and confidence intervals for the production task

\begin{tabular}{|c|c|c|c|c|c|c|c|c|c|}
\hline \multirow[b]{3}{*}{$\begin{array}{l}\text { Order of presentation/ } \\
\text { Magnitude band }\end{array}$} & \multicolumn{9}{|c|}{ Size of difference unit operand } \\
\hline & \multicolumn{3}{|c|}{ Small } & \multicolumn{3}{|c|}{ Medium } & \multicolumn{3}{|c|}{ Large } \\
\hline & $M$ & $S D$ & $95 \% \mathrm{CI}$ & $M$ & $S D$ & $95 \% \mathrm{CI}$ & $M$ & $S D$ & $95 \% \mathrm{CI}$ \\
\hline \multicolumn{10}{|c|}{ Primary 5} \\
\hline \multicolumn{10}{|l|}{ Increasing $(\mathrm{N}=55)$} \\
\hline Ones & 33 & (19) & {$[28,38]$} & 51 & (29) & {$[43,59]$} & 111 & $(56)$ & {$[95,127]$} \\
\hline Tens & 44 & $(25)$ & {$[38,51]$} & 57 & (29) & {$[49,65]$} & 116 & (59) & {$[100,132]$} \\
\hline Hundreds & 50 & (28) & {$[42,57]$} & 60 & (28) & {$[52,68]$} & 122 & (68) & {$[103,140]$} \\
\hline Thousands & 54 & $(35)$ & {$[44,63]$} & 59 & (27) & {$[52,66]$} & 106 & (62) & {$[89,123]$} \\
\hline \multicolumn{10}{|l|}{ Randomized $(\mathrm{N}=55)$} \\
\hline Ones & 35 & $(22)$ & {$[29,41]$} & 53 & (34) & {$[44,62]$} & 74 & (54) & {$[59,88]$} \\
\hline Tens & 39 & (22) & {$[33,46]$} & 54 & (26) & {$[47,61]$} & 104 & (53) & {$[89,118]$} \\
\hline Hundreds & 48 & (26) & {$[41,55]$} & 54 & (23) & {$[48,61]$} & 101 & (77) & {$[80,122]$} \\
\hline Thousands & 59 & (37) & {$[49,69]$} & 65 & $(35)$ & {$[55,74]$} & 123 & (63) & {$[106,140]$} \\
\hline \multicolumn{10}{|c|}{ Secondary 2} \\
\hline \multicolumn{10}{|l|}{ Increasing $(\mathrm{N}=36)$} \\
\hline Ones & 36 & (16) & {$[31,42]$} & 59 & (26) & {$[50,67]$} & 133 & $(57)$ & {$[114,153]$} \\
\hline Tens & 47 & (28) & {$[38,56]$} & 61 & $(25)$ & {$[53,70]$} & 124 & (51) & {$[106,142]$} \\
\hline Hundreds & 53 & (29) & {$[43,63]$} & 69 & (30) & {$[58,79]$} & 121 & (53) & {$[103,138]$} \\
\hline Thousands & 59 & (32) & {$[48,70]$} & 74 & (29) & {$[64,84]$} & 125 & (48) & {$[109,141]$} \\
\hline \multicolumn{10}{|l|}{ Randomized $(\mathrm{N}=32)$} \\
\hline Ones & 36 & (18) & {$[30,43]$} & 59 & (29) & {$[49,70]$} & 111 & (70) & {$[85,136]$} \\
\hline Tens & 52 & (25) & {$[43,61]$} & 65 & (25) & {$[56,74]$} & 138 & (73) & {$[111,164]$} \\
\hline Hundreds & 53 & (24) & {$[45,62]$} & 72 & (27) & {$[62,82]$} & 134 & (84) & {$[103,164]$} \\
\hline Thousands & 70 & (38) & {$[56,84]$} & 81 & (39) & {$[67,95]$} & 150 & (71) & {$[125,176]$} \\
\hline
\end{tabular}

Notes.

1. $M$ and $S D$ refers to the means and standard deviations of the bars drawn for the difference unit (DU).

2. $95 \% \mathrm{CI}$ refers to the $95 \%$ confidence interval. It is calculated from the sample mean and is used as an indication of the precision of the estimate. Typically, the narrower is the range, the more precise the estimate.

3. Values for the $M, S D$ and the $95 \% \mathrm{CI}$ are rounded to the nearest pixel unit.

4. Values for the DU range from 5 to 356.

Length of the difference unit drawn by the students was affected by magnitude band, $F(9,150)=$ 12.85, $p<.001, \eta_{p}{ }^{2}=.44$. As can be noted in both Table 1 and Figure 2, children generally drew longer bars for larger operands. However, this was qualified by an interaction with question sequence, $F(9,150)=4.13$, $p<.01, \eta_{p}{ }^{2}=.20$. Univariate tests showed that the interaction effect was not significant for either the smaller or medium sized operands. For these operands, there were significant and strong linear trends across the four magnitude bands, $.39>\eta_{p}{ }^{2}>.14$, regardless of sequence of presentation. In other words, children uniformly used longer bars for small and medium sized operands, regardless of magnitude band. For large operands, a strong linear and increasing trend across the four magnitude bands was found when question sequence was randomised, $F(1,80)=61.93, p<.01, \eta_{p}{ }^{2}=.44$. There were no differences in the length of the bars, across the four magnitude bands, when questions with large operand sizes were presented as part of a sequence in which the size of operands was ordered (see Figure 2). 


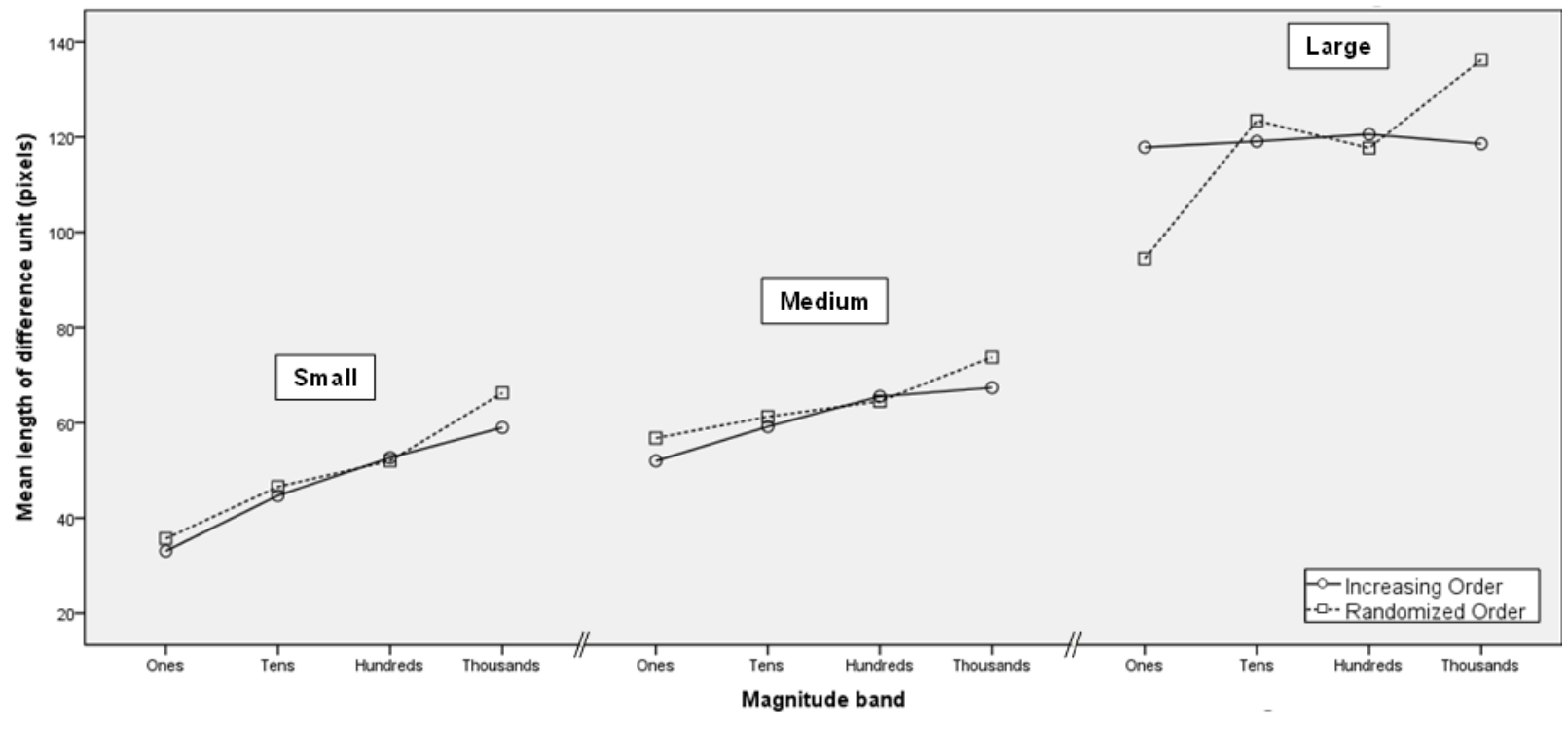

Figure 2. Mean performance on the production task by magnitude band and order of question presentation for smaller, medium and larger numbers within each band.

Although there was a significant main effect associated with grade, $F(3,156)=4.05, p<.01, \eta_{p}{ }^{2}=$ .07 , with the older children drawing longer bars, it did not enter into interaction with other variables. For Secondary 2 students, we also tested the relation between their performance on the production task and the Küchemann Algebra Test. As there were only 2 children in the Küchemann Level 1 category, Levels 1 and 2 were combined. Approximately $22 \%$ of the students attained Level 1-2, $40 \%$ Level 3, and 38\% Level 4. A 4 (magnitude: ones, tens, hundreds, vs. thousands) $\times 3$ (Küchemann: Level $1-2$, Level 3 vs. Level 4) repeated measures analysis of variance showed no significant interaction between magnitude band and level of algebraic understanding on the length of the difference unit drawn by the children. Children with more advanced understanding of the use of letters in algebra adjusted the length of bars according to magnitude in a similar manner as did students with a more basic level of algebraic understanding.

The students performed well on the validation task with $62 \%$ scoring the maximum nine marks. Oneway analysis of variance indicated that there were no significant age differences in performance on the validation task. Amongst the Secondary 2 students, performances also did not differ across levels of understanding on the Küchemann Algebra Test.

We also examined whether performance on the production task was related to performance on the validation task. Performance on the production task, relative to magnitude band, was indexed by a performance coefficient. This was derived for each individual by fitting a line of best fit using the length of their bar diagrams and the four magnitude conditions as the two axes. A larger performance coefficient indicates a greater propensity to adjust the length of the bars according to magnitude band. There was a small but significant correlation between the performance coefficient and the validation task score but only for the younger age group $(r=-0.26, p<.007)$. Primary 5 students with a higher score on the validation task were less likely to adjust the length of the bars according to the magnitude of the operands.

\section{Discussion}

Findings from the production task showed that children drew longer bars when the magnitude of the operands increased. The only condition in which children did not do this was when they were presented with larger numbers, and only when questions were presented in order of operand magnitude. These findings 
suggest that the children used the bars in a concrete fashion, but their usage is tempered by affordances in the question set. Recall that we defined concrete usage as drawing longer bars to denote larger operands, with abstract usage being indicated by the lack of a consistent relation between the length of the bars and magnitude of the operands. Children are less likely to engage in a concrete fashion when changes in the magnitude of operands across questions are more salient.

Findings from the validation task point to a different conclusion. More than half of the children scored full marks and demonstrated awareness that the absolute size of the bars were not essential to the accuracy of the model representations. In contrast to findings from the production task, this finding shows that the majority of children have quite sophisticated understanding of how the graphical representations should be interpreted. Indeed, for the younger children, there was a significant correlation between performances on the production and validation tasks. Those who showed better understanding on the validation task were more likely to produce models that conformed to our definition of abstract depiction.

Our data provide no definitive information on why the children's performance on the production task was less sophisticated than their performance on the validation task. The findings point to another case of children knowing and understanding more than what they can do. This is a common phenomenon in the development of complex skills. In the development of memory strategies, for example, children tend not to deploy skills or strategies spontaneously, but are able to do so successfully when either instructed or are given explicit prompts (Flavell, 1970; Harnishfeger \& Bjorklund, 1990). Here, both the younger and older children seem to be producing graphical depictions in a concrete manner despite having fairly sophisticated understanding. However, once the manipulation of problem size becomes apparent, they are able to deploy their knowledge accordingly. An alternative version of this explanation, which focuses on the affordances of the production task, is that the students approached the task with what is most familiar. In the production task, the children were not given specific instructions or directives on how the bar diagrams should be drawn. Although all the children have had extensive practice with the use of such graphical representations, it is possible that they drew the bars in a more concrete manner because this was what they did, and had to do, with arithmetic problems.

Findings from the validation task suggest that many children have some appreciation of the fact that the absolute length of the bars across questions is unimportant. Although this is just one aspect in understanding the role of variables in algebraic equations, from a pedagogical viewpoint, it is comforting to know that the use of the model method is not overtly associated with erroneous thinking regarding the nature of what is being represented. What is somewhat worrisome is that the secondary school children's performance on the production task was no different from the primary school children's. If the younger children's performance resulted from a production deficiency, the older children, with more experience with such questions, should have been able to use the heuristic in a more abstract fashion. Although speculative, one explanation for why this was not observed is related to the way in which algebra is taught. In secondary schools, students are taught symbolic algebra and use letter symbols to represent unknown values. In some schools at least, there is little discussion of differences and similarities in using bar diagrams versus letter symbols to represent known and unknown quantities ( $\mathrm{Ng}$, Lee, Ang, \& Khng, 2006). It is perhaps this lack of explicit linkage that resulted in some lingering confusion, which is reflected in the children's performance on the production task. Nonetheless, given their performance on both the validation task and the Küchemann test, their performance on the production task should not be viewed as a major deficit. One pedagogical approach that may further benefit student is to emphasize the different situations under which the two approaches are best suited. Although not specifically focused on the model method, previous research have shown that students are more successful when they use more concrete or grounded representations to solve simple algebra questions, but are more successful with more abstract, symbolic representations with more complex problems (Koedinger, Alibali, \& Nathan, 2008; Koedinger \& Nathan, 2004).

Findings from the Küchemann algebra test showed that the majority of our Secondary 2 students demonstrated a level of understanding that is deemed sufficient to enable them to engage in further studies in algebra. One challenging aspect of the findings is that performance on the Küchemann test was not related to performances in either the production or validation tasks. The Küchemann test focuses on how children interpret letter symbols in algebra. In contrast, our measures are focused on children's usage and understanding of the bar diagrams used to represent algebraic questions. Although an understanding of the 
use of letter symbols to represent unknowns should help children's performances in our tasks, one interpretation of the findings is that there is a lack of transfer between understanding the notion of variables when represented as letter symbols versus when represented in the form of bars. An alternative interpretation is that the two types of tasks map onto aspects of algebraic understanding that are more disparate than we anticipated. Further research on linkages between these aspects of algebra may help bridge the gap between what is taught in the primary and secondary curricula.

\section{Conclusion}

The main aim of this study was to understand how Primary and Secondary school students use and understand the bar diagrams used for solving algebraic questions. Findings from the production task showed that children generally drew longer bars for bigger operands. Although this finding shows that the children are using the graphical representations in a more concrete fashion, findings from the validation task suggest that their understanding is more sophisticated. In the validation task, both the younger and the older children demonstrated understanding that the bars can be used in an abstract manner and the length of the bars need not be tied to the size of the operands. The mismatch between findings from the production and validation tasks is interpreted as evidence of a production deficit. Although it was surprising that our Primary and Secondary school students performed in a similar fashion in the production task, when the totality of findings are considered, we do not think the evidence points to a major deficit. Despite the production finding, for the Secondary students, performances on the production and validation tasks were not correlated. Furthermore, the great majority of Secondary school students showed quite sophisticated understanding on both the validation task and the Küchemann test. On the other hand, for the Primary students, the negative correlation between the production and validation tasks suggests that examining the way these students depict problems with different operands does provide some indication of their understanding. Primary school teachers may wish to use similar sets of questions, presented in a randomised order, to gain additional insight on students' facility with algebraic concepts. It should be noted that what is important is not the length of the bars produced for each individual question, but the pattern of responses to changes in the magnitude of operands that provide insight to children's understanding. Discussing how bars of the same length can be used to represent operands of different magnitudes may also help teachers make explicit the conceptual connections between the bars and letter symbols used in algebra.

Although we were motivated by concerns regarding the role of the model method in the curriculum, this study is not an evaluation of the curriculum, nor did we evaluate whether learning the model method aids in the acquisition of letter symbolic algebra. Instead, the findings provide some answers to how children use and understand the model method, which may assist policy makers and curriculum designers when they evaluate its role in the curriculum. To that end, we were encouraged by the sophistication of the children's responses in the validation task and view these findings as being supportive of the way in which algebraic problem solving is taught.

\section{Keypoints}

- Unlike many other countries, algebraic word problems are introduced in the primary school years in the Singapore mathematics curriculum. This study examined how children understand and use bar diagrams that are used to give them earlier access to such problems.

- Both Grade 5 and 8 students showed an abstract understanding of the bar diagrams. However, they tended to use the diagrams in a more concrete fashion.

- Discrepancy between what the students produced versus what they understood is indicative of a production deficit. 
- Discussing how bars of the same length can be used to represent operands of different magnitudes in algebraic questions may help teachers make explicit the conceptual connections between the bar diagrams and letter symbols.

\section{Acknowledgements}

The work was supported by grants from the Centre for Research in Pedagogy and Practice (\#CRP 9/05 KL). Views expressed in this article do not necessarily reflect those of the National Institute of Education, Singapore. We thank the students who participated in this study and the school administrators who provided access and assistance.

\section{Appendix A}

Here are some word problems. Student A and Student B drew the models for these problems. You have to pick if Student A is correct, or Student B is correct, or if both are correct. You can do this by checking the box next to the options.

Mary has some marbles. John has 30 marbles more than Mary. They have 150 marbles altogether. How many marbles has Mary?

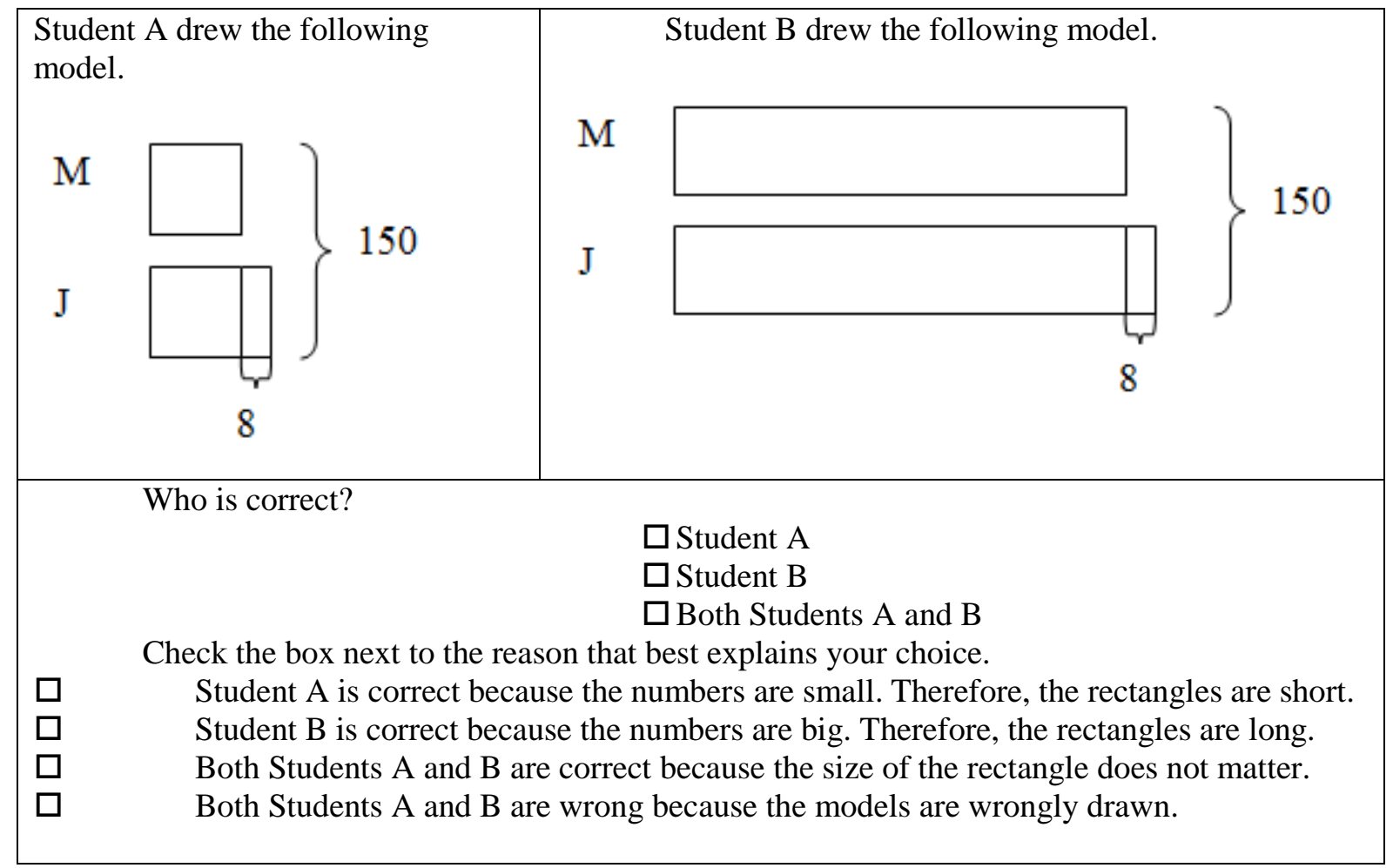

Note. One mark was awarded if the student chose "Both Students A and B" and no marks were given for choosing either "Student A" or "Student B". One mark was awarded for choosing the answer "Both Students A and B are correct because the size of the rectangle does not matter" and no marks were given for any other responses. 
Student A and Student B drew models for the 2 questions below.

Q.1

Mary has some marbles.

John has 30 marbles more than Mary.

They have 150 marbles altogether.

How many marbles has Mary?

Student A drew:

Q.1

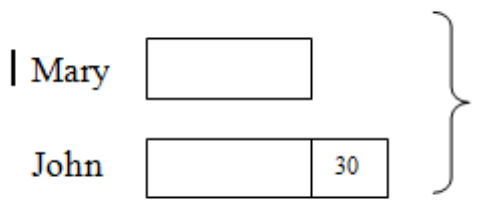

Q.2

Mary

John

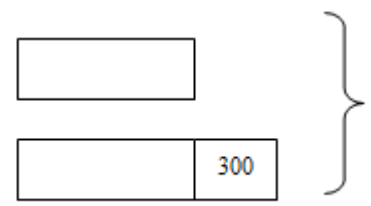

Q.2

Mary has some marbles.

John has 300 marbles more than Mary.

They have 1500 marbles altogether.

How many marbles has Mary?

Student B drew:

Q.1
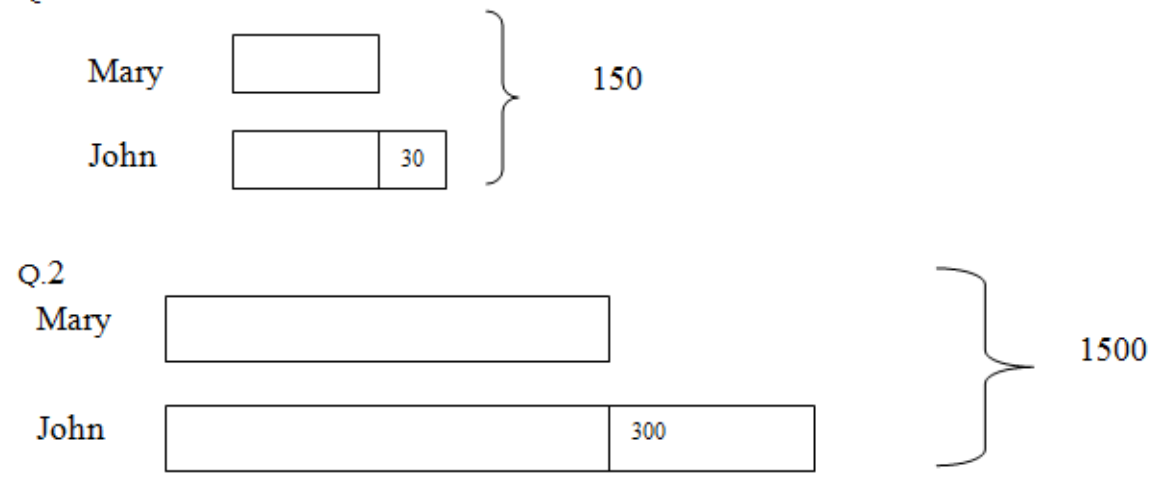

Is Student A correct?

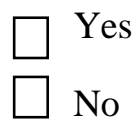

Is Student B correct?
$\square$ Yes
$\square$ No

Note. One mark was awarded for choosing "Yes" for both students and no marks were given for any other responses.

\section{References}

Akgün, L., \& Özdemir, M. E. (2006). Students' understanding of the variable as general number and unknown: A case study. The Teaching of Mathematics, 9(1), 45-51. 
Booth, J. L., \& Koedinger, K. R. (2010). Facilitating low-achieving students' diagram use in algebraic story problems. In S. Ohlsson \& R. Catrambone (Eds.), Proceedings of the 32nd Annual Meeting of the Cognitive Science Society (pp. 1649-1654). Austin, TX: Cognitive Science Society.

Brown, M., Hart, K., \& Kuchemann, D. (1985). Chelsea diagnostic mathematics tests and teacher's guide. Windsor: NFER-NELSON Publishing Company Ltd.

Capraro, M. M., \& Joffrion, H. (2006). Algebraic Equations: Can middle-school students meaningfully translate from words to mathematical symbols? Reading Psychology, 27(2-3), 147-164. doi: $10.1080 / 02702710600642467$

Carpenter, T. P., \& Levi, L. (2000). Developing conceptions of algebraic reasoning in the primary grades. (Res. Rep. 00-2). Madison, WI: National Center for Improving Student Learning and Achievement in Mathematics and Science. Retrieved from http://ncisla.wceruw.org/publications/reports/RR-002.PDF

Carraher, D. W., Schliemann, A., Brizuela, B. M., \& Earnest, D. (2006). Arithmetic and Algebra in Early Mathematics Education. Journal for Research in Mathematics Education, 37(2), 87-115.

Dede, Y. (2004). The concept of variable and identification its learning difficulties. Educational Sciences: Theory \& Practice, 4(1), 50.

Duru, A. (2011). Middle school students' reading comprehension of mathematical texts and algebraic equations. International Journal of Mathematical Education in Science and Technology, 42(4), 447468. doi: 10.1080/0020739x.2010.550938

Flavell, J. H. (1970). Developmental studies of mediated memory. In H. W. Reese \& L. P. Lipsitt (Eds.), Advances in child development and child behavior (Vol. 5). New York: Academic Press.

Fuchs, L. S., Compton, D. L., Fuchs, D., Powell, S. R., Schumacher, R. F., Hamlett, C. L., et al. (2012). Contributions of Domain-General Cognitive Resources and Different Forms of Arithmetic Development to Pre-Algebraic Knowledge. Developmental Psychology, 48(5), 1315-1326. doi: $10.1037 / \mathrm{a} 0027475$

Harnishfeger, K. K., \& Bjorklund, D. F. (1990). Children's strategies: A brief history. In D. F. Bjorklund (Eds.), Children's strategies: Contemporary views of cognitive development. Hillsdale, NJ: Lawrence Erlbaum Associates.

Hefferman, N., \& Koedinger, K. R. (1997). The composition effect in symbolizing: The role of symbol production versus text comprehension. In M. G. Shafto \& P. Langley (Eds.), Proceedings of the Nineteenth Annual Conference of the Cognitive Science Society (pp. 307-312). Mahwah, NJ: Lawrence Erlbaum Associates.

$\mathrm{Hu}$, W. (2010). Making Math Lessons as Easy as 1, Pause, 2, Pause ... The New York Times. Retrieved from http://www.nytimes.com/2010/10/01/education/01math.html?_r=0

Hunter, J. (2007). Relational or calculational thinking: students solving open number equivalence problems. In J. Watson \& K. Beswick (Eds.), Proceedings of the 30th annual conference of the Mathematics Education Research Group of Australasia (Vol. 2, pp. 421-429). Adelaide: MERGA.

Khng, K. H., \& Lee, K. (2009). Inhibiting interference from prior knowledge: Arithmetic intrusions in algebra word problem solving. Learning and Individual Differences, 19(2), 262-268. doi: 10.1016/j.lindif.2009.01.004

Kieran, C. (1981). Concepts associated with the equality symbol. Educational Studies in Mathematics, 12(3), 317-326. doi: 10.1007/bf00311062

Knuth, E. J., Stephens, A. C., McNeil, N. M., \& Alibali, M. W. (2006). Does understanding the equal sign matter? Evidence from solving equations. Journal for Research in Mathematics Education, 37(4), 297-312.

Koedinger, K. R., Alibali, M. W., \& Nathan, M. J. (2008). Trade-offs between grounded and abstract representations: Evidence from algebra problem solving. Cognitive Science: A Multidisciplinary Journal, 32(2), 366-397. doi: 10.1080/03640210701863933

Koedinger, K. R., \& Nathan, M. J. (2004). The real story behind story problems: Effects of representations on quantitative reasoning. Journal of the Learning Sciences, 13(2), 129-164.

Koedinger, K. R., \& Terao, A. (2002). A cognitive task analysis of using pictures to support pre-algebraic reasoning. In C.D. Schunn \& W. Gray (Eds.), Proceedings of the Twenty-Fourth Annual Conference of the Cognitive Science Society (pp. 542-547). Mahwah, NJ: Lawrence Erlbaum Associates. 
Küchemann, D. (1978). Children's understanding of numerical variables. Mathematics in School, 7(4), 2326.

Kwokwc. (2011). Not able to use algebra in primary level. Retrieved from http://www.kiasuparents.com/kiasu/forum/viewtopic.php?f=27\&t=18728

Lee, K., Lim, Z. Y., Yeong, S. H., Ng, S. F., Venkatraman, V., \& Chee, M. W. (2007). Strategic differences in algebraic problem solving: neuroanatomical correlates. Brain Research, 1155 (June), 163-171. doi: 10.1016/j.brainres.2007.04.040

Lee, K., Ng, S. F., Bull, R., Pe, M. L., \& Ho, R. H. M. (2011). Are patterns important? An investigation of the relationships between proficiencies in patterns, computation, executive functioning, and algebraic word problems. Journal of Educational Psychology, 103(2), 269-281. doi: 10.1037/a0023068

Lee, K., Yeong, S. H. M., Ng, S. F., Venkatraman, V., Graham, S., \& Chee, M. W. L. (2010). Computing solutions to algebraic problems using a symbolic versus a schematic strategy. ZDM, 42(6), 591-605. doi: 10.1007/s11858-010-0265-6

Lim, B. T. (2007). Can algebra be used to solve PSLE maths problems, The Strait Times. Retrieved from http://www.moe.gov.sg/media/forum/2007/forum_letters/20070217.pdf

MacGregor, M., \& Stacey, K. (1997). Students' understanding of algebraic notation: 11-15. Educational Studies in Mathematics, 33(1), 1-19. doi: 10.1023/a:1002970913563

Mayer, R. E. (1989). Systematic thinking fostered by illustrations in scientific text. Journal of Educational Psychology, 81(2), 240.

Mayer, R. E. (2002). Multimedia learning. Psychology of Learning and Motivation, 41, 85-139.

Meter, P., \& Garner, J. (2005). The Promise and Practice of Learner-Generated Drawing: Literature Review and Synthesis. Educational Psychology Review, 17(4), 285-325. doi: 10.1007/s10648-005-8136-3

Mullis, I. V. S., Martin, M. O., Gonzalez, E. J., \& Chrostowski, S. J. (2004). TIMSS 2003 International Mathematics Report: Findings From IEA's Trends in International Mathematics and Science Study at the Fourth and Eighth Grades. Chestnut Hill, MA: Boston College.

Nathan, M. J., \& Koedinger, K. R. (2000). Teachers' and researchers' beliefs about the development of algebraic reasoning. Journal for Research in Mathematics Education, 31(2), 168-190. doi: $10.2307 / 749750$

National Council for Teachers of Mathematics. (2000). Principles and Standards for School Mathematics. Reston, VA: National Council for Teachers of Mathematics.

National Mathematics Advisory Panel. (2008). Foundations for Success: The Final Report of the National Mathematics Advisory Panel. Washington, DC: U.S. Department of Education.

$\mathrm{Ng}$, S. F. (2003). How secondary two express stream students used algebra and the model method to solve problems. The Mathematics Educator, 7(1), 1-17.

$\mathrm{Ng}, \mathrm{S}$. F., \& Lee, K. (2005). How primary five pupils use the model method to solve word problems. The Mathematics Educator, 9(1), 60-84.

Ng, S. F., \& Lee, K. (2008). As Long As the Drawing is Logical, Size Does Not Matter. The Korean Journal of Thinking \& Problem Solving, 18(1), 67-82.

Ng, S. F., \& Lee, K. (2009). Model method: Singapore children's tool for representing and solving algebra word problems. Journal for Research in Mathematics Education, 40(3), 282-313.

Ng, S. F., Lee, K., Ang, S. Y., \& Khng, F. (2006). Model Method: Obstacle or bridge to learning symbolic algebra. In W. Bokhorst-Heng, M. Osborne \& K. Lee (Eds.), Redesigning Pedagogies (pp. 227-242). NY: Sense.

OECD (2010). PISA 2009 Results: Executive Summary. Retrieved from http://www.oecd.org/pisa/pisaproducts/46619703.pdf

Philipp, R. (1992). The many uses of algebraic variables. Mathematics Teacher, 85, 557-561.

Stacey, K., \& MacGregor, M. (1999). Learning the Algebraic Method of Solving Problems. The Journal of Mathematical Behavior, 18(2), 149-167.

Steinberg, R. M., Sleeman, D. H., \& Ktorza, D. (1991). Algebra students' knowledge of equivalence of equations. Journal for Research in Mathematics Education, 22(2), 112-121.

Swafford, J. O., \& Langrall, C. W. (2000). Grade 6 students' preinstructional use of equations to describe and represent problem situations. Journal for Research in Mathematics Education, 31(1), 89-112. 
Tolar, T. D., Lederberg, A. R., \& Fletcher, J. M. (2009). A structural model of algebra achievement: computational fluency and spatial visualisation as mediators of the effect of working memory on algebra achievement. Educational Psychology: An International Journal of Experimental Educational Psychology, 29(2), 239-266.

Usiskin, Z. (1988). Conceptions of school algebra and uses of variables. In A. F. Coxford \& A. P. Schulte (Eds.), The Ideas of Algebra (pp. 8-19). Reston, VA: National Council of Teachers of Mathematics.

Warren, E., \& Cooper, T. (2005). Introducing functional thinking in year 2: a case study of early algebra teaching. Contemporary Issues in Early Childhood, 6(2), 150-162.

Warren, E., \& Cooper, T. J. (2009). Developing mathematics understanding and abstraction: the case of equivalence in the elementary years. Mathematics Education Research Journal, 21(2), 76-95.

Wei, W., Yuan, H. B., Chen, C. S., \& Zhou, X. L. (2012). Cognitive correlates of performance in advanced mathematics. British Journal of Educational Psychology, 82(1), 157-181. doi: 10.1111/j.20448279.2011.02049.x 